\title{
Neanderthals built underground
}

The finding of 175,000-year-old structures deep inside a cave in France suggests that Neanderthals ventured underground and were responsible for some of the earliest constructions made by hominins.

\section{MARIE SORESSI}

$\mathrm{B}$ uilding is a frequent by-product of human activity, and some ancient constructions remain majestic to this day. However, all too often, constructions made by mobile populations do not preserve well. Evidence for structures made by prehistoric hunter-gatherers are scarce and usually consist only of an area of finds with intriguing spatial distributions, which may be associated with a fireplace. In a paper online in Nature, Jaubert et al. ${ }^{1}$ report the discovery of circular structures made of broken stalagmites deep inside a cave in southwest France. The structures are up to 40 centimetres high and 6.7 metres wide, and direct radiometric dating shows that they are at least 175,000 years old. Because Neanderthals were the only hominin group present in western Europe at that time, the discovery provides the first directly dated evidence for Neanderthals' construction abilities. It also shows that Neanderthals explored underground.

Neanderthals lived in Eurasia from around 400,000 to 40,000 years ago, at which point anatomically modern humans settled in. Investigation of the archaeological record from the Late Pleistocene epoch - which spanned from 126,000 to 11,700 years ago - has provided robust data on the behaviour of ancient hominins and allowed a comparison of the activities of Neanderthals and early modern humans. This comparative approach has been regularly used to elaborate on the reasons for Neanderthals' demise and the success of early modern humans.

However, given a lack of direct evidence, there has been little discussion of the constructional abilities of Neanderthals. It is known that great apes, birds and other animals build elaborate nests (the bowerbird is a famous example), and the archaeological record contains examples of constructions made by anatomically modern humans about 20,000 years ago, such as collapsed, rounded 'ruins' made from mammoth bones or deer antlers ${ }^{2}$. Yet only a few structures interpreted as post-holes or isolated elements of dry stone walls have been tentatively attributed to Neanderthals. Furthermore, differential distributions of finds inside and outside potential Neanderthal constructions have rarely been documented, and even then not always convincingly ${ }^{2}$.

Jaubert et al. report accumulations of almost 400 stalagmites and stalagmite fragments stacked into several structures, including two that have a semicircular shape, some $300 \mathrm{~m}$ from the entrance of Bruniquel Cave (Fig. 1). One semicircular structure, which is more than $6.7 \mathrm{~m}$ wide, comprises a 'wall' made of up to four superimposed layers of stalagmite fragments about $30 \mathrm{~cm}$ in length, with smaller elements stuck obliquely in between. Reddening, blackening and cracking of many stalagmites suggest that the structures have been heated by small fires. The authors also recovered a $6.7-\mathrm{cm}$ fragment of heated bone from within one of the smaller structures, close to reddened and blackened stalagmites.

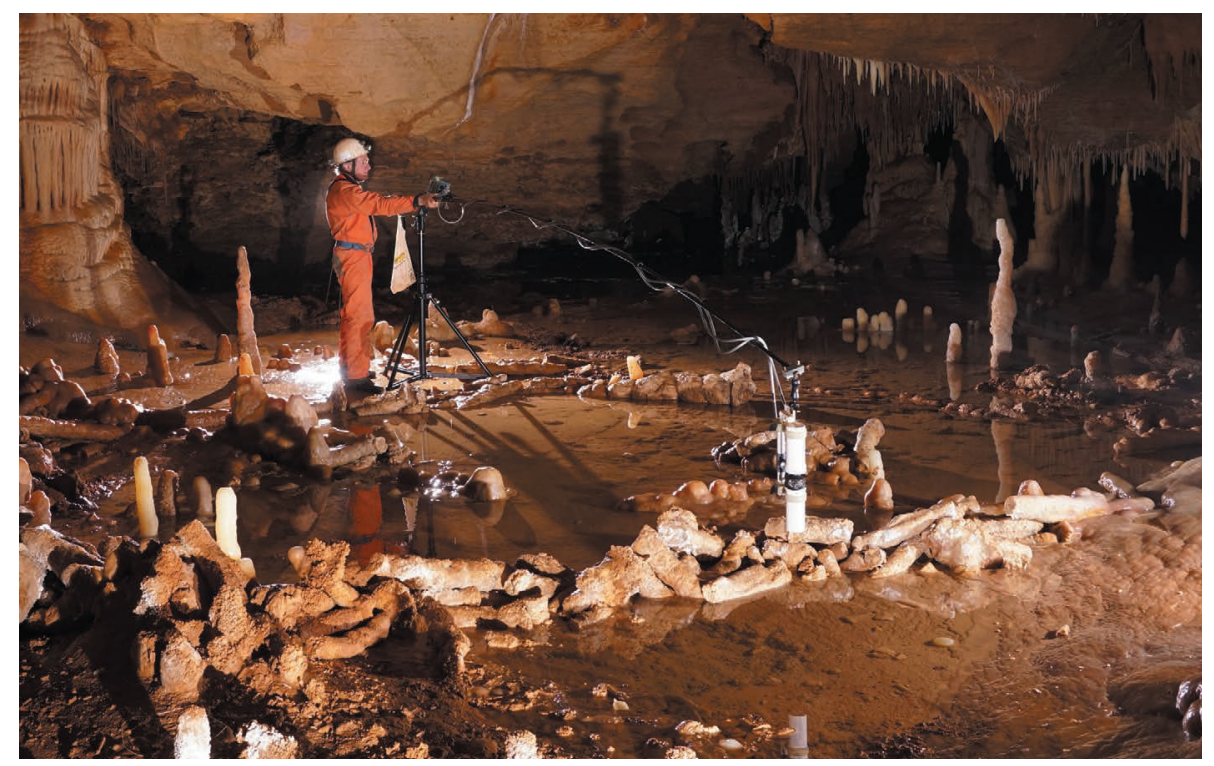

Figure 1 | Ancient structures. Circular structures made from broken stalagmites, found in Bruniquel Cave in southwest France by Jaubert et al. ${ }^{1}$, are thought to have been made by Neanderthals around

175,000 years ago.

This find, together with measurement of the magnetic anomalies in the rock above and around the structures, supports the idea that the structures were heated.

The researchers used molecular and atomic spectrometry to investigate two other probable residues of heated bones, one found in a 2-m-wide structure and the other forming part of a concentration of similarly blackened material discovered on the ground and interpreted as a hearth. Seven stalagmites from the two largest structures were dated using uranium-series measurements; by dating the calcite that had grown before and after the fragments were broken, the researchers could constrain the date at which the stalagmites were used in construction. The calcite covering the $6.7-\mathrm{cm}$-long bone and forming the flowstone (a sheet-like calcite deposit) on the floor of the largest structure was also dated.

Altogether, the authors dated 18 samples from the area containing the structures, which show that the structures are around 176,500 years old (with a confidence interval of 2,100 years). That period is known to have had relatively warm and humid phases, which is consistent with the calcite deposition observed. The signal of oxygen and carbon isotopes reported from the stalagmites is also consistent with the atmospheric conditions known for that time.

The inner organization and the size of the structures do not fit with what is documented for the nests of cave bears, discounting that possibility for their construction. Thus, these structures are the oldest directly dated 
constructions attributed to Neanderthals, and the first ones for which we can be confident of that attribution. Furthermore, no charred materials have been found outside the structure, and no red- or black-coloured material was observed on the cave ceiling above the structure: these details support the idea that the colorations are indicative of heating in situ and were not transported between or onto the stalagmites by natural processes.

Jaubert et al. discuss the social organization that would have been needed to manufacture such structures, and compare this with what is known for modern humans from the same era. They conclude that their discovery indicates that Neanderthals exhibited morecomplex social behaviour than was previously thought, and suggests that these hominins used the underground environment. Only further discovery of underground structures will help to establish whether these structures were opportunistic ones relating to an accidental underground visit, or whether they were part of regular and planned Neanderthal activities.

These structures are among the best- preserved constructions known for the whole of the Pleistocene epoch, probably because they were sealed by calcite very soon after they were erected. When the best evidence is found in the best-preserved context, it serves as a reminder for archaeologists of how much we depend on preservation. The fact that some of the art of the period is also often found deep inside caves has been alternatively interpreted as a testimony of the preservation provided by the cave environment ${ }^{3}$ or as a result of spiritual preoccupations - the underground being a special place ${ }^{4}$. Perhaps we need to further consider the idea that the fuzziness of the Neanderthal record is due to a lack of preservation. Given that we often discuss archaeological findings in a comparative framework that contrasts Neanderthals (which disappeared) with early modern humans (who were obviously successful), we may also wonder how this framework is biased by Western thought. European culture is known for having emphasized what may be 'uniquely human' and may separate 'us' from other animals.

Comparing hominins across a large chunk of time is necessary and useful. However, an increased focus on reconstructing the historical context of past behavioural and technological innovations may be key to further understanding these different populations. The structures discovered by Jaubert et al. are a good example of how reconstructing ancient history may benefit from not only broad-scale comparisons of evolution over time but also detailed analysis of specific areas at specific time points.

Marie Soressi is in the Faculty of Archaeology, Leiden University, 2300 RA Leiden, the Netherlands.

e-mail:m.a.soressi@arch.leidenuniv.nl

1. Jaubert, J. et al. Nature http://dx.doi.org/10.1038/ nature18291 (2016) (2016)

2. Klein, R. G. The Human Career: Human Biological and Cultural Origins 3rd edn (Univ. Chicago Press, 2009).

3. Guthrie, R. D. The Nature of Paleolithic Art (Univ. Chicago Press, 2005).

4. Clottes, J. \& Lewis-Williams, J. D. Les Chamanes de la Préhistoire: Transe et Magie dans les Grottes Ornées: Suivi de Après les Chamanes, Polémiques et Réponses (Seuil, 1996). 